\title{
Dust Particle Artifact Detection and Removal in Retinal Images
}

\section{Detección y eliminación de artefactos de partículas de polvo en imágenes de la retina}

\author{
E. Sierra ${ }^{1}$, A. G. Marrugo ${ }^{1 *}$, M. S. Millán ${ }^{2, S}$ \\ 1. Facultad de Ingeniería, Universidad Tecnológica de Bolívar, Cartagena, Colombia. \\ 2. Departament d'Òptica i Optometria, Universitat Politècnica de Catalunya

\begin{abstract}
:
Retinal fundus cameras suffer from dust particles attaching to the sensor and lens, which manifest as small artifacts on the images. We propose a new strategy for the detection and removal of dust particle artifacts in retinal images. We consider as input two or more color fundus images acquired within the same session, in which we assume the artifacts remain in the same position. Our method consists in detecting candidate artifacts via normalized cross correlation with an artifact template, performing segmentation via region growing, and comparing the segmentations in all images. This guarantees that all detections are consistent for all images. The removal stage consists in an inpainting procedure so that the new region does not stand out from the neighboring regions. Encouraging experimental results show the localization of artifacts is effective and the artifacts are successfully removed, while not introducing new artifacts in the color retinal images.
\end{abstract}

Key words: dust particle artifacts, retinal image, inpainting, artifact localization, retinal image enhancement.

\section{RESUMEN:}

Las cámaras de fondo de ojo sufren de partículas de polvo que se adhieren al sensor y la lente. Éstas aparecen en las imágenes como pequeños artefactos. En este trabajo proponemos una nueva estrategia para la detección y eliminación de artefactos en imágenes de fondo ojo producidos por partículas de polvo. Se consideran como entrada dos o más imágenes de fondo de ojo a color adquiridas en la misma sesión; se asume que los artefactos no cambian de posición. El método propuesto consiste en la detección de artefactos mediante la correlación cruzada normalizada con una plantilla de artefactos, realizar la segmentación por crecimiento de regiones y comparar las segmentaciones en todas las imágenes. Esto garantiza que las detecciones sean consistentes para todas las imágenes. La etapa de eliminación consiste en un procedimiento de inpainting de tal manera que la nueva región no resalte respecto de las regiones vecinas. Los resultados experimentales han sido satisfactorios en los cuales se muestra que la localización de artefactos es efectiva y los artefactos se eliminan satisfactoriamente sin introducir nuevos artefactos en las imágenes retinianas a color.

Palabras clave: imagen retiniana, artefactos en imágenes, detección de artefactos, eliminación de artefactos.

\section{REFERENCES AND LINKS / REFERENCIAS Y ENLACES}

[1] M. D. Abramoff, M. Garvin, and M. Sonka, "Retinal Imaging and Image Analysis," Biomedical Engineering, IEEE Reviews in, 3, 169-208 (2010).

[2] R. G. Willson, M. Maimone, A. Johnson, and L. Scherr, An optical model for image artifacts produced by dust particles on lenses. 2005, 1-8. 
[3] C. Zhou and S. Lin, "Removal of Image Artifacts Due to Sensor Dust," 1-8, Apr. 2007.

[4] A. D. Mora, J. Soares, and J. M. Fonseca, "A template matching technique for artifacts detection in retinal images," presented at the 2013 8th International Symposium on Image and Signal Processing and Analysis (ISPA), 717-722.

[5] M. Niemeijer, M. D. Abramoff, and B. van Ginneken, "Image structure clustering for image quality verification of color retina images in diabetic retinopathy screening," Medical Image Analysis, 10, 888898 (2006).

[6] A. G. Marrugo, M. S. Millan, G. Cristóbal, S. Gabarda, and H. C. Abril, "No-reference Quality Metrics for Eye Fundus Imaging," CAIP 2011, LNCS, 6854, 486-493 (2011).

[7] T. Köhler, A. Budai, M. Kraus, J. Odstrcilik, G. Michelson, and J. Hornegger, "Automatic no-reference quality assessment for retinal fundus images using vessel segmentation.," presented at the IEEE 26th International Symposium on Computer-Based Medical Systems (CBMS), 2013, 95-100.

[8] S. A. Ali Shah, A. Laude, I. Faye, and T. B. Tang, "Automated microaneurysm detection in diabetic retinopathy using curvelet transform," J. Biomed. Opt., 21, 101404 (2016).

[9] P. Yang, L. Chen, J. Tian, and X. Xu, "Dust particle detection in surveillance video using salient visual descriptors," Computers \& Electrical Engineering, 2016.

[10] L. Chen, D. Zhu, J. Tian, and J. Liu, "Dust particle detection in traffic surveillance video using motion singularity analysis," Digit Signal Process, 1-7 (2016).

[11] A. G. Marrugo, E. Sierra, and M. S. Millan, "Dust Particle Detection and Correction in Retinal Images," presented at the RIAO-OPTILAS 2016, Pucón, Chile, 268, 2016.

[12] R. Radke, S. Andra, O. Al-Kofahi, and B. Roysam, "Image change detection algorithms: a systematic survey," Image Processing, IEEE Transactions on, 14, 294-307 (2005).

[13] A. G. Marrugo, M. Sorel, F. Sroubek, and M. S. Millan, "Retinal image restoration by means of blind deconvolution," J. Biomed. Opt., 16, 116016 (2011).

[14] A. G. Marrugo and M. S. Millan, "Retinal Image Analysis Oriented to the Clinical Task," Electronic Letters on Computer Vision and Image Analysis, 13, 54-55 (2014).

[15] R. C. Gonzalez, R. E. Woods, and S. L. Eddins, Digital Image Processing Using MATLAB®. McGraw Hill Education, 2010.

[16] D.-M. Tsai and C.-T. Lin, "Fast normalized cross correlation for defect detection," Pattern Recognition Letters, 24, 2625-2631 (2003).

[17] A. G. Marrugo and M. S. Millan, "Optic disc segmentation in retinal images," Opt. Pura Apl., 43, 79-86 (2010).

[18] A. Neubeck and L. Van Gool, "Efficient non-maximum suppression," presented at the Pattern Recognition, 2006. ICPR 2006. 18th International Conference on, 2006, 3, 850-855.

[19] R. M. Haralick, S. R. Sternberg, and X. Zhuang, "Image analysis using mathematical morphology," Pattern Analysis and Machine Intelligence, IEEE Transactions on Pattern Analysis and Machine Intelligence, 9, 532-550 (1987).

\section{Introduction}

Retinal images are a useful tool for medical diagnosis since many diseases, not just ocular ones, are manifested in the retina by signs or anomalies [1]. Retinal fundus cameras, just like any imaging device suffer from dust particles attaching to the sensor and lens. These particles manifest as small artifacts or blemishes on the images, which may hinder its diagnostic purpose. For example, these artifacts can be mistaken as small lesions, such as micro-aneurysms.

Sensor and lens dust are a common problem in digital photography. The dust particles (including atomized liquid stains) accumulate and block incoming light to the sensor. They produce image artifacts that typically appear as dark spots on the image. These artifacts and those produced by defective pixels in an image sensor, the so-called dead pixels, have similar effects. Since these defective pixels are permanent, some manufacturers provide their cameras with image processing algorithms such as the nearest neighbor algorithm applied to analog and digital video for bad pixel remapping. The problem with dust becomes more serious because it is unavoidable in common clinical practice and accumulative. This alteration of the captured image, in particular in retinal images, is critical to their clinical value. Sensor cleaning is possible, but not often done because it requires professional assistance and time lapses of inactivity. Therefore, many images are usually acquired before the sensor is cleaned or the technician notices the artifacts.

The effect of dust particles in imaging has been described by Wilson et al. [2] but their work mainly addresses the modeling of the dust particles attaching to the lens and not their detection or removal. Zhou 
and Lin [3], propose a dust artifact formation model and a detection and removal approach based on an optimization scheme. They assume that the dust particle is made of a single material, its projection onto the sensor should appear approximately monochromatic, and it should have color properties different than the surrounding region. Despite the fact that they succeed in detecting the majority of artifacts in their tests, their approach is mostly applicable to natural scenes.

Artifact detection in retinal images has been mostly oriented toward imaging artifacts such as flash flares [4], image quality verification [5]-[7] or as a previous stage for automated diabetic retinopathy screening [8]. However, the detection and removal of image artifacts from sensor dust in retinal images has not been studied extensively. More recently several works for dust particle image artifact detection in video sequences have been proposed on the assumption that the scene changes in time but not the location of the artifacts [9], [10]. Our approach is based on this idea of several input images and the artifacts remaining in approximately the same location.

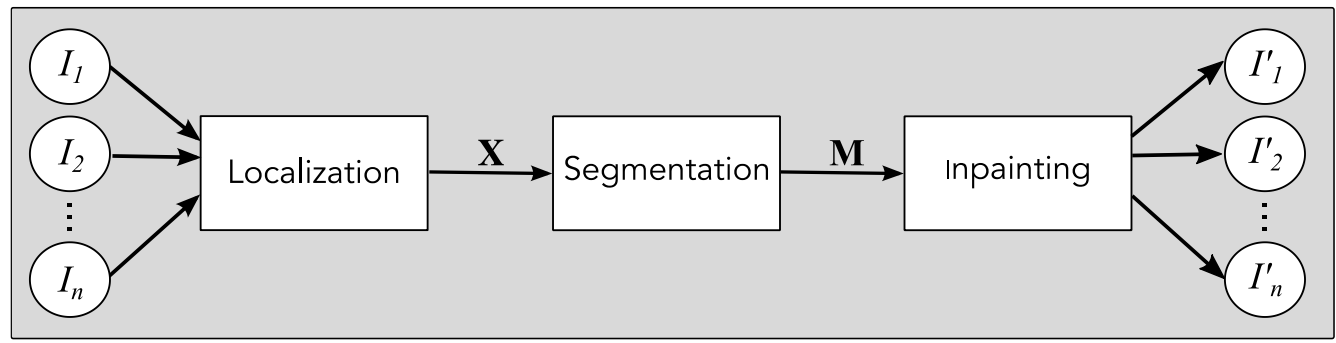

Fig.1. Block diagram illustrating the proposed method. $I_{i}$ are the input images, $I_{i}^{\prime}$ are their restored versions. The other variables are intermediate outputs of every stage; their meaning is given in the text.

\section{1.a. Overview of the proposed approach}

In this paper we propose a new strategy for the detection and removal of dust particle artifacts in retinal images [11]. We assume that the image artifacts do not change position within several acquisitions taken from a given patient during their visit, for instance the first acquisition corresponding to the left eye fundus and the second acquisition to the right eye fundus. Hence, we can formulate an approach that compares detection based on two or more images. Furthermore, because of the medical importance of the images, it is desirable to have a low false-positive rate. In other words, the algorithm should not detect artifacts that are not actually artifacts. A micro-aneurysm by no means should be detected as an artifact. In this regard, the algorithm should be highly specific in not altering regions void from artifacts. With the use of two or more images for comparing detection results, the algorithm is less prone to false detections.

An overview of the proposed approach is described in Fig. 1. We consider as input two or more color fundus images acquired with a conventional fundus camera within the same session, typically left and right eye fundus from the same patient. The images have artifacts produced from dust particles in the sensor. These are approximately in the same location in the image. Therefore, the first stage is to detect candidate artifacts and obtain their coordinates, which are stored in the matrix $\boldsymbol{X}$. The second stage consists in segmenting these candidate artifacts, which is carried out via region growing and comparing the segmentations with a conjunction or logical AND operation. This guarantees that all detections are consistent throughout all images [12]-[14]. The segmentation output is a binary matrix $\boldsymbol{M}$ with ones in every pixel corresponding to a detected artifact and zeros elsewhere. Finally, the removal of the image artifacts is carried out by an inpainting procedure, which consists in smoothly interpolating from the boundaries of the artifact in such a way that the new region does not stand out from the neighboring regions, i.e. no new artifacts are introduced. This does not mean that the inpainted area would be replaced by the corresponding part of the ideal image. We must be aware that it would remain as part of the problem uncertainty, but at least, this part would appear camouflaged by the surrounding area and would not convey misleading information that could be mistakenly interpreted. 


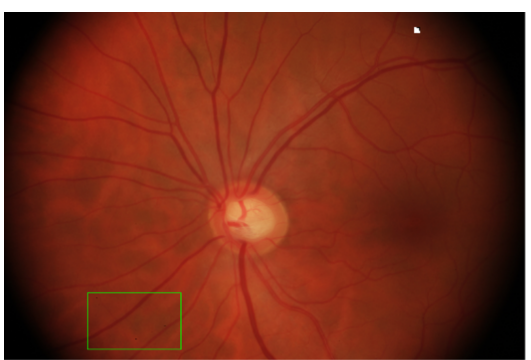

(a)

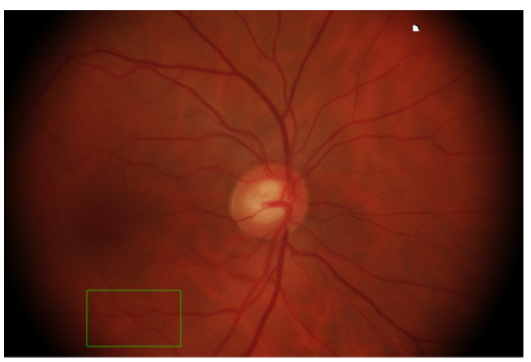

(c)

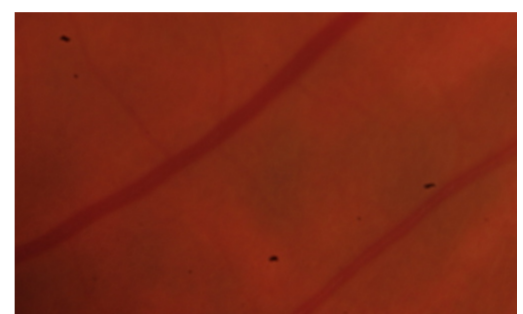

(b)

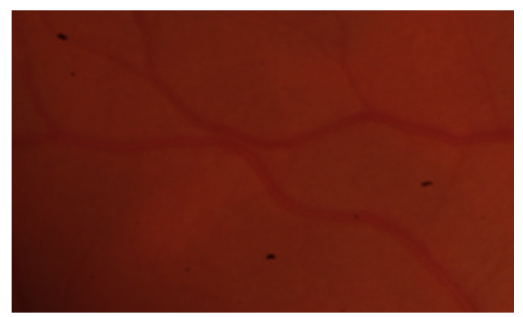

(d)

Fig.2. (a) and (c) color retinal images taken from the same patient, one of each eye. (b) and (d) zoomed regions of (a) and (c) respectively, where presence of artifacts is observed.

\section{Description of the Method}

In this section, we describe every stage of the proposed method. To illustrate each stage we use the images shown in Fig. 2. These correspond to two images from the same patient acquired at the same session but from different eyes, therefore they are different images. However, notice how the artifacts (dark spots) are approximately in the same position, regardless of the image content. They are color RGB 24 bit-depth fundus images of size $2784 \times 1846$ digitized in JPEG format. The localization and segmentation stages are carriedout processing the images in gray-scale (a weighted sum of the R, G, B components), but the inpainting procedure and final output images are in color.

\section{2.a. Localization}

Localization of artifacts in images may be regarded as a blob detection task, common in pattern recognition, in that several elements of the images have a similar structure and need to be located and possibly segmented [15]. A common approach to blob detection in pattern recognition is template matching via cross correlation. This operation determines the position of a given template $t$ in an image $f$ by calculating the similarity of the template to all points within the image. Cross-correlation is calculated as [16]

$$
c(u, v)=\sum_{x, y} f(x, y) t(x-u, y-v) .
$$

The cross-correlation achieves a maximum value in the positions where the template exactly matches a region in $f$. However, cross correlation has a clear disadvantage. If the image energy varies with position, the correlation between the template and a bright area will give a high value as result [16]. Therefore, template matching using equation (1) often fails. The problem can be overcome by normalizing both the image and the template. This leads to the normalized cross-correlation (NCC) [16]

$$
\gamma(u, v)=\frac{\sum_{x, y} f(x, y) t(x-u, y-v)}{\sqrt{\sum_{x, y}\left[f(x, y)-\bar{f}_{u, v}\right]^{2} \sum_{x, y}[t(x-u, y-v)-\bar{t}]^{2}}},
$$

where $\bar{t}$ is the mean of the template $t$ and $\bar{f}$ is the mean of $f$ in the region under the template. The NCC returns values in the interval $[-1,1]$ where 1 means highest correlation. To localize the blobs we used the NCC, given by equation (2), of the images with an artifact template. As is often done for optic nerve head 
loalization [17], we built the template by manually segmenting four artifacts chosen arbitrarily and averaging them (Fig. 3).

Template matching does not imply perfect correlation, therefore we threshold the NCC output at $90 \%$ of the maximum value to identify the regions where the template best matches the image. To identify local maxima, we perform non-maximum suppression [18] to obtain the candidate artifacts coordinates.

In Fig. 4 we show the procedure of applying the normalized cross-correlation between two input images and the artifact template. The coordinates of the candidate artifacts obtained via non-maximum suppression are stored in matrix $\boldsymbol{X}_{\boldsymbol{i}}$, for the $\boldsymbol{i}$-th retinal image. The blob detections for the images of Fig. 2 are shown in Fig. 5. In Fig. 5(a), we show the actual artifacts marked with arrows (ground truth). In Fig. 5(b) we show the blob detection results, which are shown in green for the true positives and in blue for the false positives. When compared with the actual artifacts, notice how we are able to successfully detect the most salient artifacts, while the missed artifacts are smaller and more transparent.

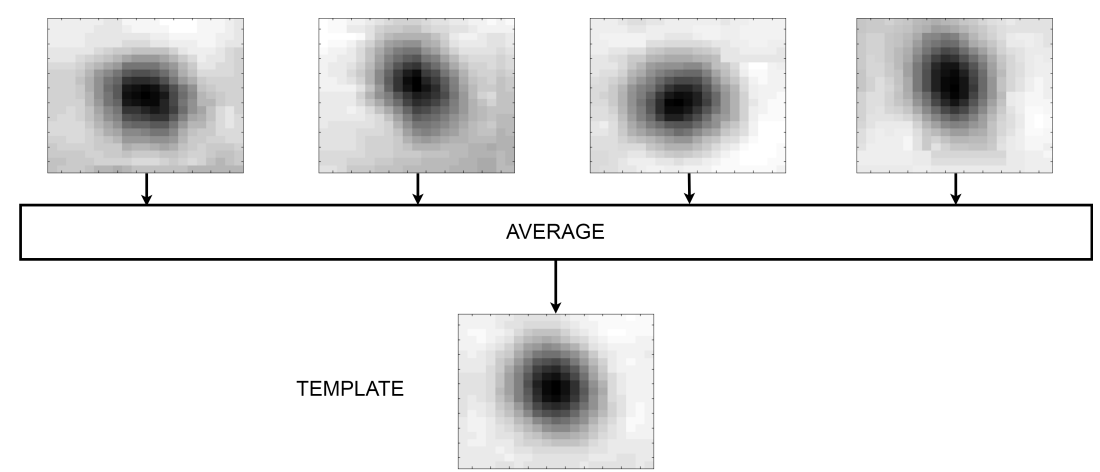

Fig. 3. The artifact template was built by averaging several artifacts.

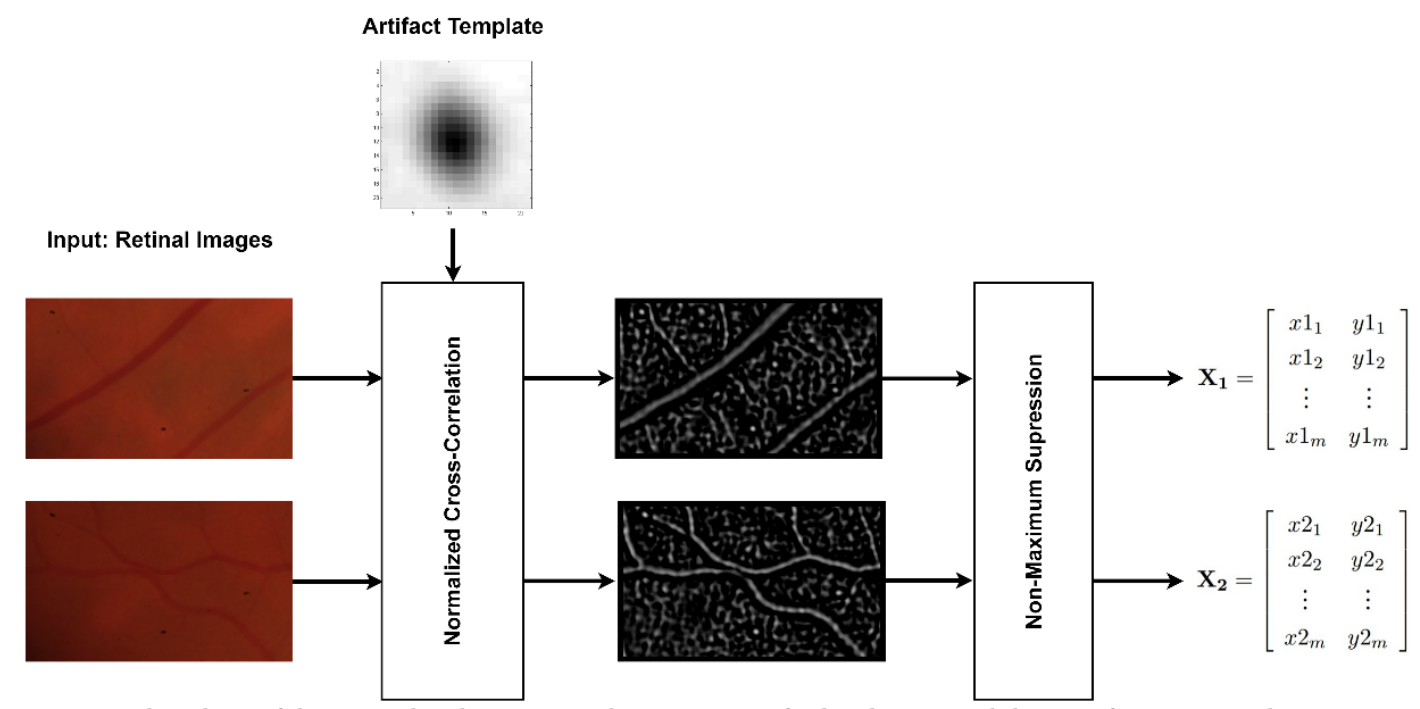

Fig.4. Flowchart of the normalized cross-correlation process for localizing candidate artifacts in retinal images.

\section{2.b. Segmentation}

After the localization stage, the artifacts have to be segmented for successful removal. For the segmentation procedure we use a region-growing algorithm [15]. The region-growing algorithm starts with a set of seed pixels, from which regions are created. First of all, a seed pixel is chosen and it is compared with its neighboring pixels. Those that comply with predefined criteria of growth are added to the region. The region grows by adding new neighboring pixels that are similar to those that are already part of the region. When the growth of the region stops, the algorithm chooses another seed pixel that does not belong to any region and starts again. The algorithm enforces the following criteria to add a pixel to a region: i) it must be a neighbor of at least one pixel that is already part of the region and ii) the difference of intensity values between the two pixels must not exceed a set threshold [15]. 
We apply the region-growing algorithm on the NCC result. The coordinates in $\boldsymbol{X}$ from the localization stage are used as seeds. The result from the region-growing algorithm is a binary mask $\boldsymbol{M}$. These masks correspond to the blobs detected as potential artifacts for each input image as shown in Fig. 6.

It is important to mention that false alarms or false positives appear. In order to eliminate them, we take into account that the artifacts are in the same positions in all the images. Therefore, the blobs that actually correspond to artifacts should be present in every mask $\boldsymbol{M}$ for each image. These masks are compared by a logic AND operation in order to eliminate false alarms. The resulting mask is obtained with the blobs that are common to all input images. The final mask is dilated with a circular structuring element to obtain a slightly larger segmentation area for the following inpainting procedure [19]. Notice that in the blob detection shown in Fig. 5, two of the smallest artifacts are correctly detected in the bottom image (Fig. 5(d)), but because they are not detected in the upper image they will not classify as artifacts. There are also false positives, which are eliminated with this procedure.

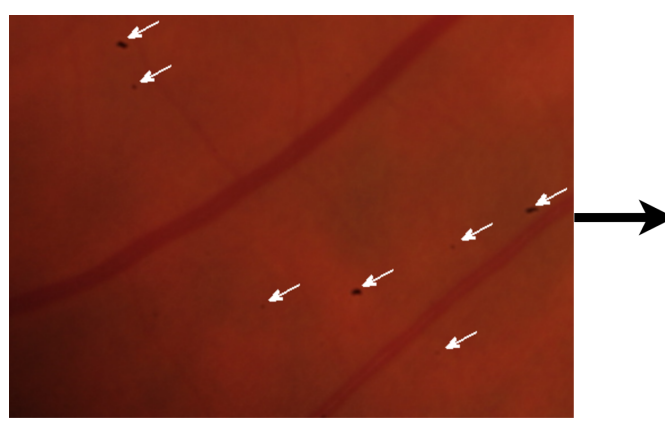

(a)

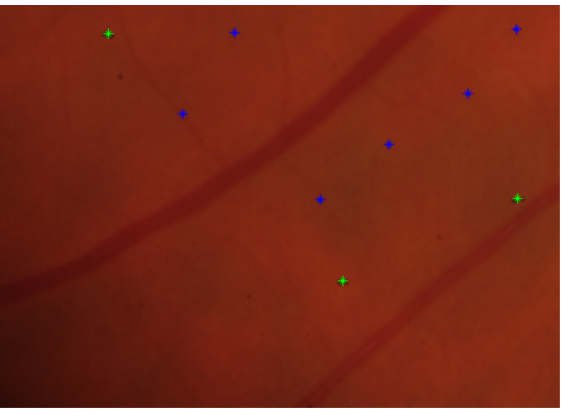

(b)

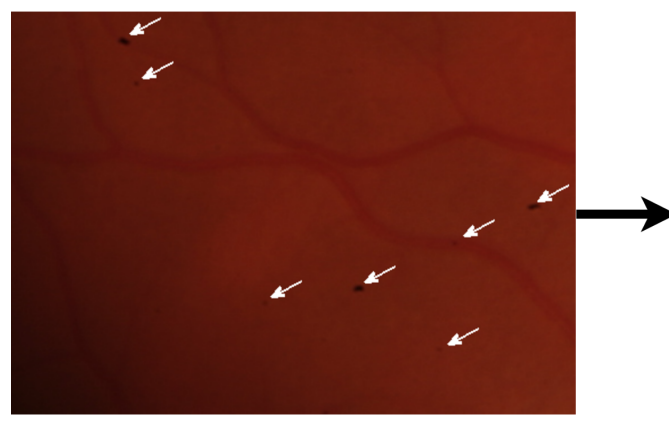

(c)

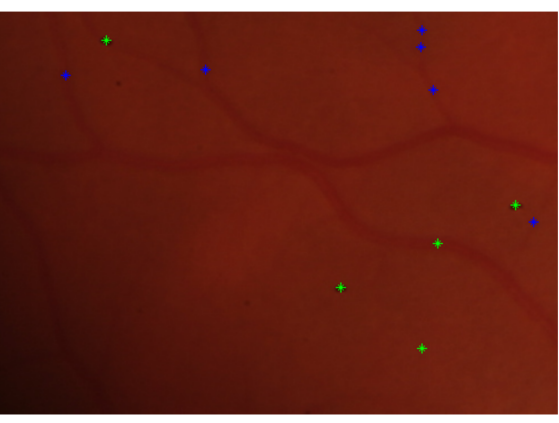

(d)

Fig.5. Ground truth: (a) and (c) show the images of Fig. 2 (b) and (d), respectively, with arrows pointing out the actual artifacts. (b) and (d) show the NCC output for potential artifact location, green dots indicate the true positives and blue dots the false positives.

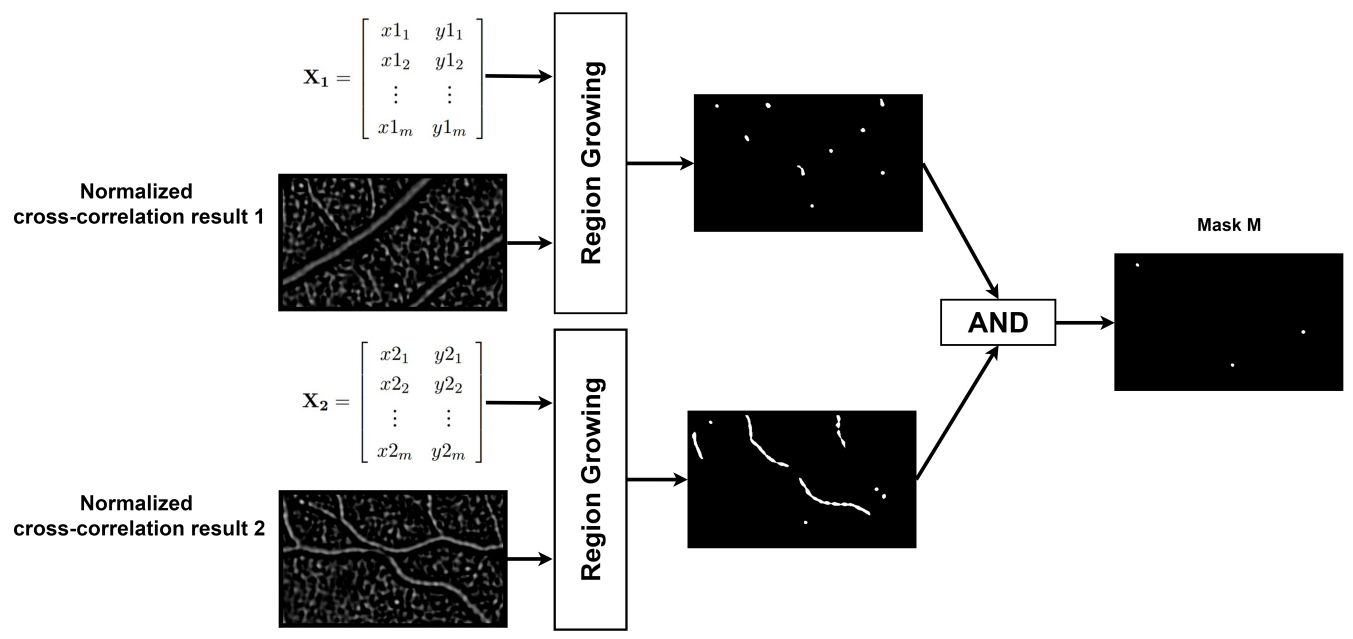

Fig.6. Flowchart to obtain the mask of detected artifacts. 


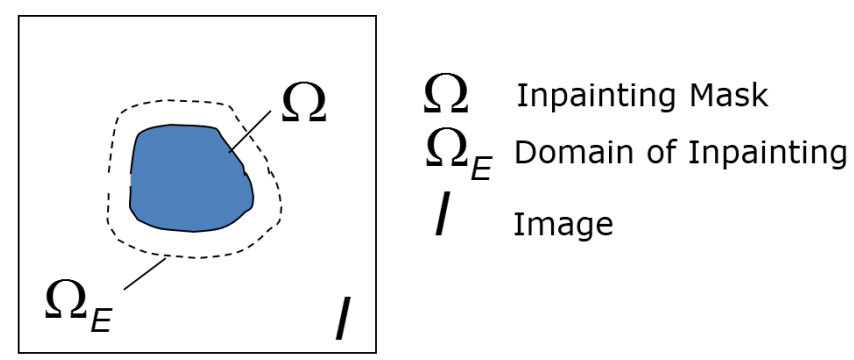

Fig.7. Image region considered for inpainting.

\section{2.c. Inpainting}

The problem of artifact removal or inpainting can be considered as follows: given an image $I$ and a region $\Omega$ inside it, modify the values of $\Omega$ from the surrounding area $\Omega_{E}$, in such a way as to maintain the texture and structure of the surroundings (Fig. 7).

There are different ways to approach the problem of inpainting since it is a difficult problem and the formulation depends on the type of image of interest. The method we propose here is based on a discretized solution of the Laplace equation with boundary conditions, given by

$$
\nabla^{2} f=0
$$

where

$$
\nabla^{2} f=\frac{\partial^{2} f}{\partial x^{2}}+\frac{\partial^{2} f}{\partial y^{2}} .
$$

Operating this way a smooth interpolation is obtained along the region of interest, allowing us to obtain a texture similar to that of the surrounding region. The value of one pixel in $\Omega$ is replaced by the average of the north, south, east and west neighbors that are not in $\Omega$. This operation is performed from the edge of $\Omega$ towards the center, without changing the values of the pixels that surround the region.

The artifact masks $\boldsymbol{M}$ are the actual inpainting masks used to perform the removal of artifacts. An important advantage of the mask $\boldsymbol{M}$ is that it can be further used for removing artifacts in all images that were acquired in the same session and thus have the same artifacts in the same positions. In other words, the mask can be built from a few images, but many can be further processed with the resulting mask.

The image artifacts are removed by processing the image in sub-windows centered at the image artifact coordinates. This makes the process suitable for parallel processing. Since the input images are RGB color images, the inpainting is carried out in each color channel separately to reduce color artifacts in the retinal image.

\section{Results and Discussion}

In this section we show the experimental results obtained from restoring real retinal fundus images affected with dust particle artifacts. We carried out four tests of retinal image pairs that made a total of eight images to check the performance and efficiency of the algorithm.

In order to properly show the results, the figures show only the region of the retinal images affected with artifacts. In Fig. 8 we show a successful detection and removal of all artifacts. Notice the quality of the restoration in which the inpainted regions are not noticeable. In Fig. 9 we show an example of a dark region from a retinal image in which seven out of the eight artifacts were successfully detected and removed. The artifact at the bottom of the image was not detected. The proposed algorithm is able to detect and remove artifacts in dark areas without introducing new artifacts.

Fig. 10 (a) shows an image with six artifacts (ground truth). Most artifacts are correctly detected and removed without introducing new artifacts (fig. 10b), however the three smallest artifacts with low contrast were not detected. These small artifacts with low contrast are much more difficult to detect. Actually, it requires a trained eye to identify them as artifacts. They become evident when comparing several images. 
The code was implemented in MATLAB on a PC running windows 7 Intel i5 8 GB RAM. Processing two $2784 \times 1846$ color retinal images takes on average 20 seconds.

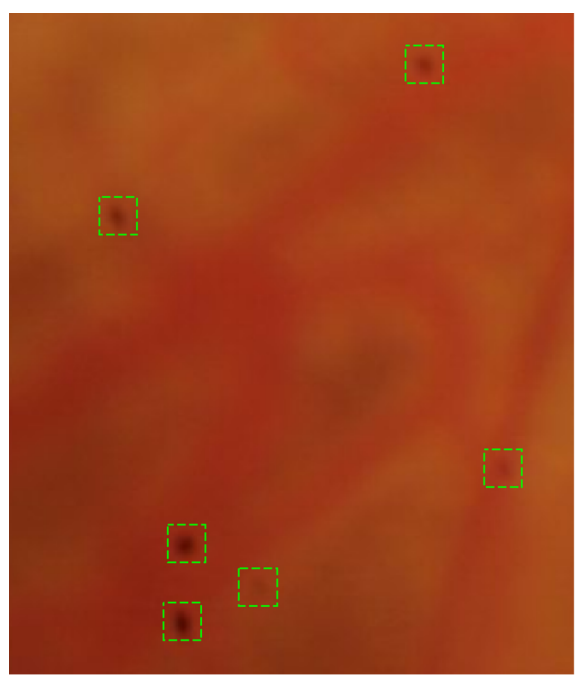

(a)

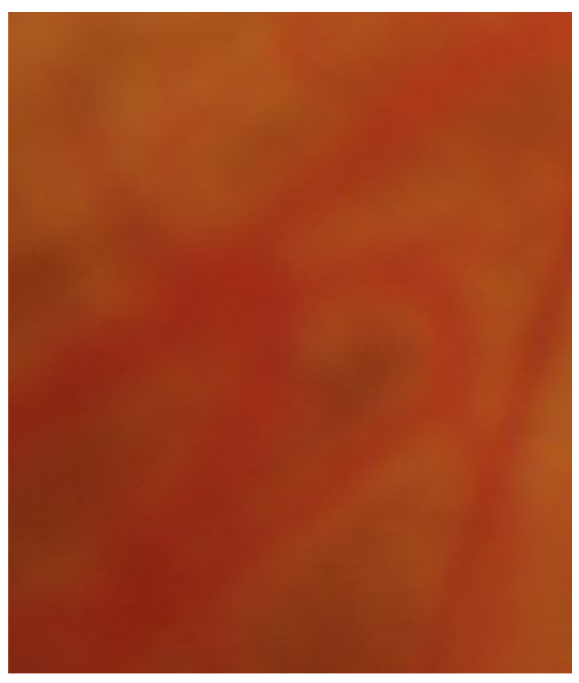

(b)

Fig.8. (a) image window with detected artifacts prior to inpainting. (b) Result after inpainting.

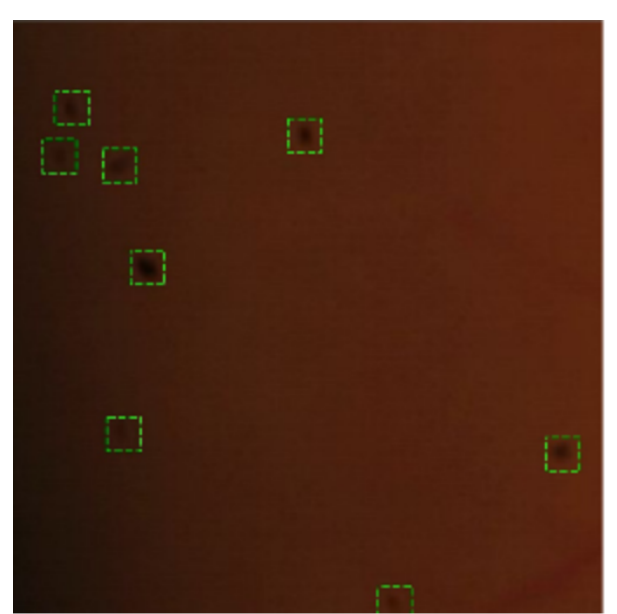

(a)

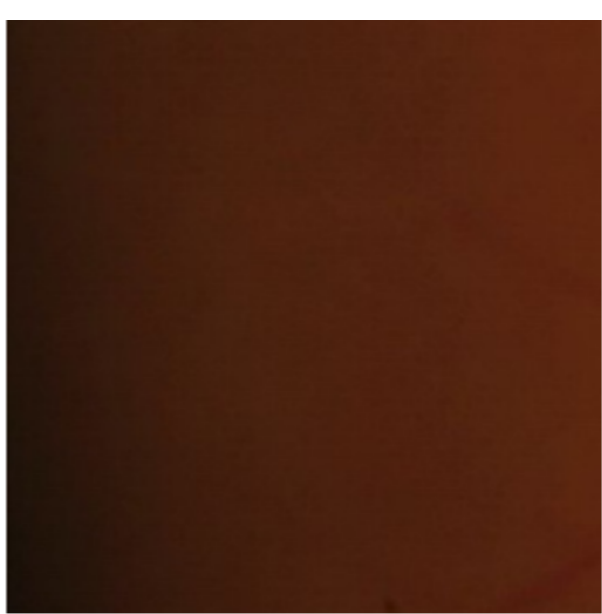

(b)

Fig.9. (a) image window with detected artifacts prior to inpainting. (b) Result after inpainting.

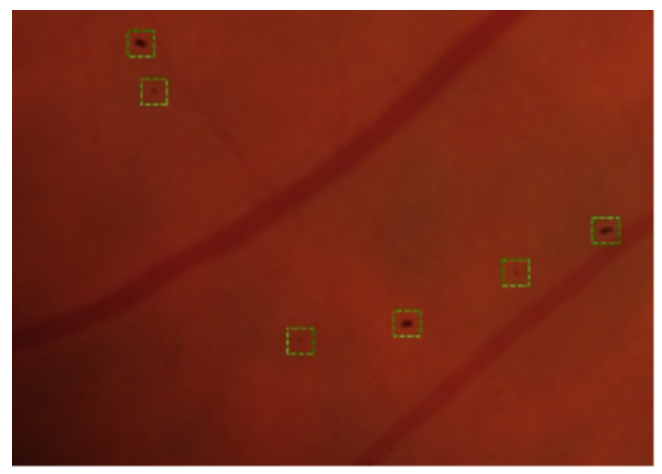

(a)

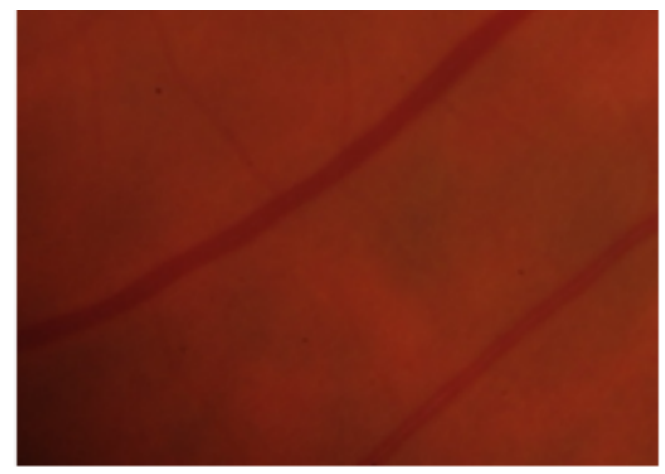

(b)

Fig.10. (a) image window with detected artifacts prior to inpainting. (b) Result after inpainting. 


\section{Conclusions}

In this work we proposed a method for detecting and removing dust particle artifacts that appear as dark spots in retinal fundus images. Although the inpainted regions still remain as a part of the problem uncertainty, the remapped spots would no longer stand out among the surrounding areas thus avoiding the possibility of being mistakenly interpreted as signs of pathological risk.

The localization of artifacts is effective, it succeeds in finding the majority of artifacts in the retinal images. The false alarms are minimized by the AND-comparison of the artifact detections and segmentations from several input images. This gives robustness to the algorithm. The segmentation stage determines the region or group of pixels of the potential artifacts. In our experiments, the inpainting approach removed successfully the artifacts, while not introducing new artifacts in the color retinal images. The code is sufficiently fast, and is suitable for parallelization, which can significantly reduce computation time.

\section{Acknowledgements}

This research has been partly funded by the Spanish Ministerio de Economía y Competitividad y Fondos FEDER (project No. DPI2016-76019-R) and and by the Centre de Cooperació i Desenvolupament de la Universitat Politècnica de Catalunya BARCELONATECH (project No. 2017-U009). The first author thanks the Universidad Tecnológica de Bolívar for a Masters Degree scholarship. 